\title{
The Association between Serum Uric Acid and Residual $\beta$-Cell Function in Type 2 Diabetes
}

\author{
Wei Tang, ${ }^{1}$ Qi Fu, ${ }^{2}$ Qingqing Zhang, ${ }^{2}$ Min Sun, ${ }^{2}$ Yuan Gao, ${ }^{1}$ \\ Xuan Liu, ${ }^{2}$ Li Qian, ${ }^{2}$ Shan Shan, ${ }^{2}$ and Tao Yang ${ }^{2}$ \\ ${ }^{1}$ Department of Endocrinology and Metabolism, The Affiliated Jiangyin Hospital of Southeast University Medical College, \\ Jiangyin, Jiangsu 214400, China \\ ${ }^{2}$ Department of Endocrinology, The First Affiliated Hospital of Nanjing Medical University, Nanjing, Jiangsu 210029, China
}

Correspondence should be addressed to Wei Tang; drtangwei@aliyun.com and Tao Yang; yangt@njmu.edu.cn

Received 16 November 2013; Revised 14 April 2014; Accepted 7 May 2014; Published 26 May 2014

Academic Editor: Li Yanbing

Copyright (C) 2014 Wei Tang et al. This is an open access article distributed under the Creative Commons Attribution License, which permits unrestricted use, distribution, and reproduction in any medium, provided the original work is properly cited.

\begin{abstract}
The aim of this study was to investigate the relationship of serum uric acid (sUA) with residual $\beta$-cell function in type 2 diabetes. Oral glucose tolerance tests (OGTT) were performed on 1021 type 2 diabetes patients. The ratio of area under curve of insulin to glucose during 0 to $30 \mathrm{~min}$ and 0 to $120 \mathrm{~min}$ of the OGTT was calculated as indices of insulin secretion function. The products of insulin secretion indices multiplied by Matsuda insulin sensitivity index were used as disposition indices. After correlation and multiple linear regression analysis, sUA was significantly associated with insulin secretion and disposition indices in male, female, and total groups adjusted for confounding factors (including metabolic indicators like sex, age, course of the disease, blood glucose, blood pressure, serum lipids, and so on). Superficially steeper time-dependent decline of insulin secretion function was found in patients with sUA above the median than those below it. In conclusion, our results suggest an independent positive association between sUA and residual $\beta$-cell function in type 2 diabetes. Patients with higher sUA have greater insulin secretion ability than those with lower sUA at the early stage of disease, but their residual $\beta$-cell function seems to decay more rapidly.
\end{abstract}

\section{Introduction}

Lots of studies found increased serum uric acid (sUA) levels in subjects with metabolic syndrome (MetS) or cardiovascular disease, and sUA is associated with several components of MetS, including dyslipidemia, hypertension, impaired glucose metabolism, and obesity [1-3]. Some recent studies have already highlighted the connection between sUA and glucose homeostasis. For example, the Rotterdam Study showed that the subjects with higher levels of sUA were at higher risk of type 2 diabetes [4], and a modest positive association between plasma uric acid concentration and the incidence of type 2 diabetes in Chinese individuals was suggested by Chien et al. [5]. As is known, both insulin resistance and $\beta$-cell dysfunction play determinate roles in the pathogenesis of type 2 diabetes [6]. The correlation between sUA and insulin resistance has been verified by several researches years ago $[7,8]$, and insulin resistance is thought to be the principal underlying pathophysiologic abnormality connecting hyperuricemia and components of MetS. However, most previous studies were performed on nondiabetic subjects. Furthermore, few studies focused on the relationship between sUA level and islet $\beta$-cell dysfunction, and whether there is a corresponding change of sUA level with $\beta$-cell function deterioration in type 2 diabetes is unknown. In this cross-sectional study, we investigated the association between sUA and $\beta$-cell function as well as insulin sensitivity in patients with type 2 diabetes, and we further elucidate the time-dependent changes of insulin secretion ability in different gender and uric acid level groups.

\section{Materials and Methods}

2.1. Study Subjects. One thousand and twenty-one patients with type 2 diabetes who received treatment in the First Affiliated Hospital of Nanjing Medical University between 2008 and 2011 were enrolled in this study. Type 2 diabetes mellitus 


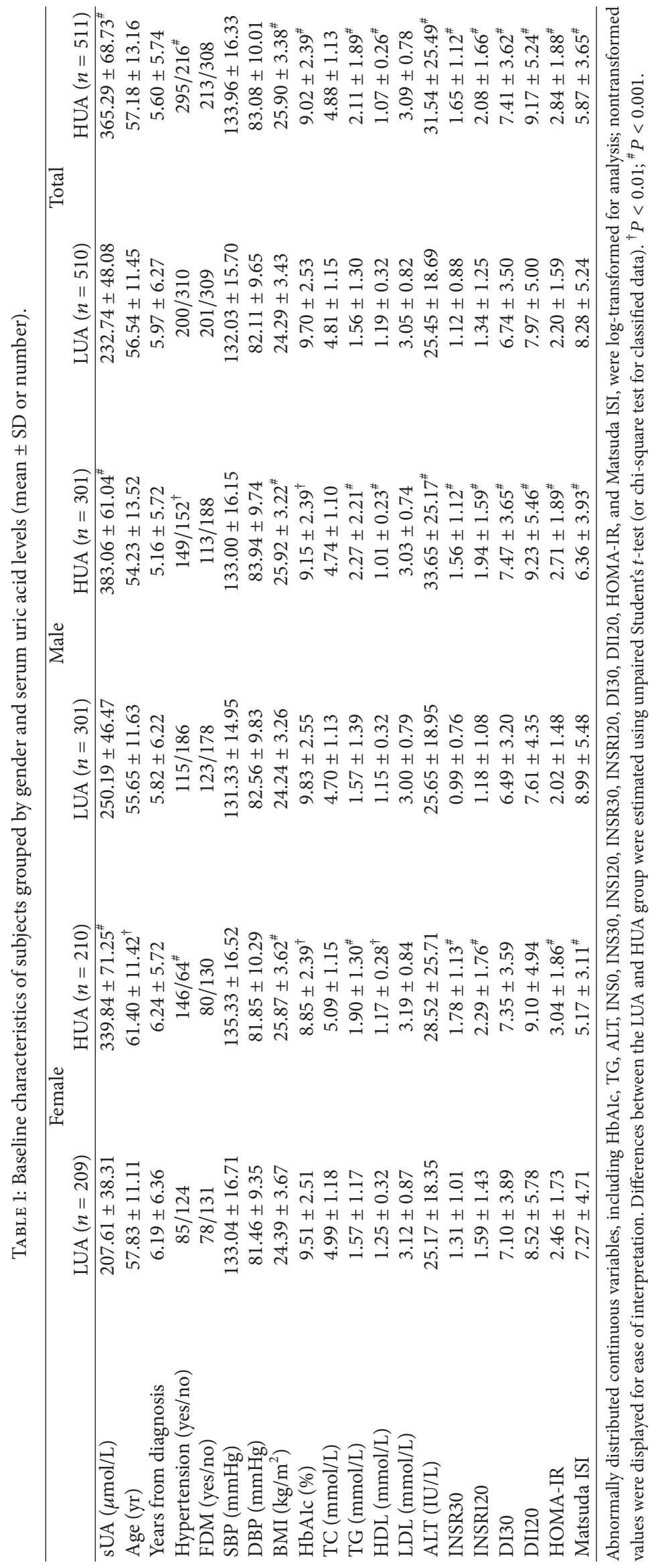


was diagnosed according to the criteria of the American Diabetes Association [9]. The maximum duration of diabetes was 35 years. The average age of all patients was $56.86 \pm 12.34$ (mean \pm SD) years old. Meanwhile, body mass index (BMI) and sUA were $25.10 \pm 3.50 \mathrm{~kg} / \mathrm{m}^{2}$ and $299.08 \pm 88.96 \mu \mathrm{mol} / \mathrm{L}$, respectively. Patients with severe pancreatic disease, liver disease, and renal disease and those who suffered recent diabetic ketoacidosis and hyperosmotic nonketotic diabetic coma were excluded. Verbal informed consent was obtained from all participants. The study was approved by the ethics committee of the First Affiliated Hospital of Nanjing Medical University.

2.2. Measurements. In all subjects, the height, weight, systolic blood pressure (SBP), and diastolic blood pressure (DBP) were measured and recorded. History of hypertension, family history of diabetes (FHD), and the years from diagnosis of type 2 diabetes were inquired. If one of parents, siblings, or grandparents had been diagnosed with diabetes, patient was defined as having FHD. All patients stopped using antidiabetic medicine at least one day before the blood samples are taken. After 10-12 hours overnight fasting, venous blood samples were collected to measure uric acid, glycated haemoglobin (HbAlc), triglyceride (TG), total cholesterol (TC), high-density lipoprotein cholesterol (HDL), low-density lipoprotein cholesterol (LDL), alanine aminotransferase (ALT), fasting plasma glucose (G0), and fasting serum insulin (I0). Then the $75 \mathrm{~g}$ oral glucose tolerance test (OGTT) and insulin secretion test were performed, and venous blood samples were obtained at 30 and 120 minutes after glucose load for measuring the plasma glucose (G30, G120) and serum insulin (I30, I120).

Plasma glucose concentrations were measured using the hexokinase method (OLYMPUS AU5400). HbAlc and serum insulin were measured by high performance liquid chromatography (Bio-Rad D10) and radioimmunoassay (Iodine $\left[{ }^{125} \mathrm{I}\right]$ Insulin Radioimmunoassay Kit, Beijing North Institute of Biological Technology), respectively. ALT and serum lipid profiles, including TG, HDL, and LDL, were determined with an automatic biochemical analyzer (HITACHI 7020).

2.3. Calculations. BMI was calculated through dividing weight $(\mathrm{kg})$ by square of height $(\mathrm{m})$. To evaluate the insulin secretion, InsAUC30/GluAUC30 (INSR30) was calculated as a surrogate index for the early phase insulin secretion and InsAUC120/GluAUC120 (INSR120) as a surrogate index for total insulin secretion, where InsAUC30 and GluAUC30 are the area under insulin (mIU/L) and glucose $(\mathrm{mmol} / \mathrm{L})$ curves during 0 to $30 \mathrm{~min}$ of the OGTT and InsAUC120 and GluAUC120 are the area under insulin and glucose curves during 0 to $120 \mathrm{~min}$, respectively [10]. Matsuda insulin sensitivity index (Matsuda ISI, calculated as $10000 / \sqrt{(\mathrm{G} 0 \times \mathrm{I} 0) \times(\overline{\mathrm{G}} \times \overline{\mathrm{I}})}$, where $\overline{\mathrm{G}}$ and $\overline{\mathrm{I}}$ are the average levels of plasma glucose $(\mathrm{mg} / \mathrm{dL})$ and insulin $(\mathrm{mIU} / \mathrm{L})$ during OGTT) was chosen to evaluate insulin sensitivity [11]. The homeostasis model assessment of insulin resistance (HOMAIR) index was calculated as follows: insulin $(\mathrm{mIU} / \mathrm{L}) \times$ glucose $(\mathrm{mmol} / \mathrm{L}) / 22.5$ [12]. Glucose disposition indices (disposition
TABLE 2: Correlations between serum uric acid level and $\beta$-cell function as well as insulin sensitivity.

\begin{tabular}{lccc}
\hline & Total & Female & Male \\
& $r$ & $r$ & $r$ \\
\hline INSR30 & $0.301^{\#}$ & $0.290^{\#}$ & $0.395^{\#}$ \\
INSR120 & $0.296^{\#}$ & $0.275^{\#}$ & $0.393^{\#}$ \\
DI30 & $0.150^{\#}$ & $0.100^{*}$ & $0.206^{\#}$ \\
DI120 & $0.166^{\#}$ & $0.115^{*}$ & $0.226^{\#}$ \\
HOMA-IR & $0.192^{\#}$ & $0.216^{\#}$ & $0.241^{\#}$ \\
Matsuda ISI & $-0.252^{\#}$ & $-0.261^{\#}$ & $-0.336^{\#}$ \\
\hline
\end{tabular}

All abnormally distributed continuous variables were log-transformed. ${ }^{*} P<$ $0.05 ;{ }^{\#} P<0.001$.

index $30=$ DI30 $=$ Matsuda ISI $\times$ INSR30, disposition index $120=$ DI120 = Matsuda ISI $\times$ INSR120 $)[10,13]$ were used to assess $\beta$-cell function, combining both insulin secretion and insulin sensitivity.

2.4. Statistical Analysis. Student's $t$-test and the chi-square test were used to analyze group differences. The correlativity between sUA and $\beta$-cell function was analyzed by Pearson's correlation. The multiple linear regression analysis was applied to test the associations between $\beta$-cell function and sUA after adjustment for several covariates. Abnormally distributed continuous variables, including HbAlc, TG, and ALT, as well as all the indices for insulin sensitivity and $\beta$ cell function, were log-transformed to yield an approximately normal distribution before statistical analysis. $P$ value $<0.05$ (two-tailed) was considered statistically significant. All statistical analyses were conducted with the Statistical Package for Social Science for Windows (SPSS, version 13.0).

\section{Results}

The characteristic of the study patients was shown in Table 1 . The patients were divided into two groups according to the median sUA levels of females and males, respectively (LUA: low serum uric acid, which was under the median sUA level; HUA: high serum uric acid, which was above the median sUA level). Patients with HUA had higher levels of BMI, TG, and ALT and greater ratio of hypertension in both genders. In contrast, the HbAlc and HDL were lower in HUA groups than in LUA ones. In male, female, and total groups, HUA patients had greater HOMA-IR and smaller Matsuda ISI than LUA subjects. Consistent with insulin resistance, patients with HUA had greater insulin secretion indices, that is, INSR30 and INSR120, than LUA in all groups. Interestingly, we found that in men and total groups, patients with HUA had greater disposition indices (both DI30 and DI120) rather than in women group. Statistically significant correlations were found between sUA level and all the indices of $\beta$-cell function and insulin sensitivity either totally or after being stratified by gender (shown in Table 2).

To exclude confounding factors which may influence $\beta$ cell function, multiple linear regression was carried out using all clinical parameters which were significantly correlated 
TABLE 3: Multiple linear regression for $\beta$-cell function in total patients.

\begin{tabular}{|c|c|c|c|c|c|c|c|c|}
\hline & \multicolumn{2}{|c|}{ INSR30 } & \multicolumn{2}{|c|}{ INSR120 } & \multicolumn{2}{|c|}{ DI30 } & \multicolumn{2}{|c|}{ DI120 } \\
\hline & $\beta$ & S $-\beta$ & $\beta$ & S- $\beta$ & $\beta$ & $\mathrm{S}-\beta$ & $\beta$ & $\mathrm{S}-\beta$ \\
\hline Sex $(0=$ female, $1=$ male $)$ & -0.201 & $-0.136^{\#}$ & -0.229 & $-0.139^{\#}$ & - & - & - & - \\
\hline Hypertension $($ yes $=1$, no $=0)$ & -0.026 & -0.018 & -0.018 & -0.011 & - & - & - & - \\
\hline Years from diagnosis & -0.020 & $-0.165^{\#}$ & -0.029 & $-0.218^{\#}$ & -0.016 & $-0.217^{\#}$ & -0.026 & $-0.287^{\#}$ \\
\hline $\mathrm{BMI}\left(\mathrm{kg}^{2} / \mathrm{cm}\right)$ & 0.021 & $0.005^{\#}$ & 0.018 & $0.076^{\dagger}$ & - & - & - & - \\
\hline $\mathrm{SBP}(\mathrm{mmHg})$ & - & - & - & - & 0.002 & 0.055 & 0.001 & 0.021 \\
\hline $\mathrm{DBP}(\mathrm{mmHg})$ & - & - & - & - & -0.005 & $-0.099^{\dagger}$ & -0.006 & $-0.111^{\#}$ \\
\hline HbAlc (\%) & -0.116 & $-0.400^{\#}$ & -0.156 & $-0.479^{\#}$ & -0.075 & $-0.414^{\#}$ & -0.114 & $-0.527^{\#}$ \\
\hline $\mathrm{sUA}(\mu \mathrm{mol} / \mathrm{L})$ & 0.001 & $0.147^{\#}$ & 0.001 & $0.146^{\#}$ & 0.001 & $0.154^{\#}$ & 0.001 & $0.156^{\#}$ \\
\hline TG $(\mathrm{mmol} / \mathrm{L})$ & -0.006 & -0.005 & 0.015 & 0.012 & - & - & - & - \\
\hline $\mathrm{TC}(\mathrm{mmol} / \mathrm{L})$ & - & - & - & - & -0.011 & -0.029 & -0.019 & -0.040 \\
\hline $\mathrm{HDL}(\mathrm{mmol} / \mathrm{L})$ & -0.077 & -0.032 & -0.098 & -0.036 & - & - & - & - \\
\hline $\mathrm{LDL}(\mathrm{mmol} / \mathrm{L})$ & - & - & - & - & -0.007 & -0.012 & 0.009 & 0.014 \\
\hline ALT (IU/L) & 0.091 & $0.076^{\dagger}$ & 0.103 & $0.077^{\dagger}$ & - & - & - & - \\
\hline HOMA-IR & 0.497 & $0.455^{\#}$ & 0.426 & $0.349^{\#}$ & -0.222 & $-0.326^{\#}$ & -0.279 & $-0.343^{\#}$ \\
\hline
\end{tabular}

B: partial regression coefficient; S- $\beta$ : standard partial regression coefficient. All abnormally distributed continuous variables were log-transformed. Only parameters which were independently associated with $\beta$-cell function indices after multiple stepwise regression are shown. ${ }^{\dagger} P<0.01 ;{ }^{\#} P<0.001$.

with $\beta$-cell function indices (data of correlation was not shown) as independent variables and indices of $\beta$-cell function as dependent variables. The results of multiple linear regression analysis are shown in Table 3 . The sUA was independently associated with INSR30 and INSR120 ( $P$ value of both partial regression coefficients was less than 0.001) after adjustment for sex, hypertension, years from diagnosis, BMI, HbAlc, TG, HDL, ALT, and HOMA-IR. In the regression model of DI30 and DI120, the relationship between sUA and disposition indices was statistically significant $(P$ value of both partial regression coefficients was less than 0.001 ) after adjustment for years from diagnosis, SBP, DBP, HbAlc, TC, LDL, and HOMA-IR. After grouping patients by sex, the regression models of $\beta$-cell function were shown in Table 4. Serum uric acid remained significantly associated with insulin secretion indices (INSR30 and INSR120) and disposition indices (DI30 and DI120) after adjusting for potential confounding factors in both female and male groups (all $P$ value of partial regression coefficients was less than 0.05).

We also investigated the differences of $\beta$-cell function changes along with disease duration between low and high sUA levels. In the scattered plots displayed in Figures 1 and 2, in both females and males, INSR30 and INSR120 decreased along with disease duration in both HUA and LUA group (all regression coefficients were negative, $P<0.05$ ). Interestingly, although INSR30 and INSR120 were higher in HUA group at the early stage of diabetes, they superficially decreased more rapidly along with disease duration than in LUA group and finally dropped to almost the same level as the LUA group. However, the difference of slop of the lines was not statistically significant after adjusting for confounding factors including hypertension, BMI, HbAlc, TG, HDL, ALT, and HOMA-IR. For disposition indices, in HUA group, DI30 and DI120 significantly dropped as disease duration increased in both females and males. However, in LUA group, no significant regression was found between disposition indices and disease duration in both genders, except for DI120 in females.

\section{Discussion}

The failure of pancreatic $\beta$-cell function plays an important role in the pathogenesis of type 2 diabetes. Previous studies have shown that impaired insulin secretion is the key in the conversion from normal glucose tolerance (NGT) to impaired glucose tolerance (IGT) and diabetes $[14,15]$, and the deterioration of $\beta$-cell function does not stop after diagnosis. The United Kingdom Prospective Diabetes Study (UKPDS) showed that $\beta$-cell function, assessed by homeostasis model assessment (HOMA), decreased approximately by $25 \%$ in the first 5 years of diabetes [16]. Several factors, including hyperglycemia, dyslipidemia, cytokines secreted by adipocytes, and immune response, have been proposed as reasons for pancreatic $\beta$-cell function deterioration [1719]. In addition, our study found the close relationship between sUA and insulin secretion ability as well as glucose disposition indices, which was rarely concerned in previous studies.

Concerning the relationship between sUA level and two critical sides in the pathogenesis of type 2 diabetes, that is, insulin resistance and $\beta$-cell dysfunction, the interrelationship between sUA and insulin resistance was revealed by several studies years ago $[7,8]$. Our present study verified this relationship again by calculating the insulin sensitivity by either Matsuda ISI or HOMA-IR. Elevated sUA level usually accompanies insulin resistance. Higher insulin levels can reduce renal excretion of urate and enhance renal urate reabsorption with increased renal tubular reabsorption of sodium $[7,20,21]$. In addition, increased purine biosynthesis and turnover, with its attendant increase in sUA, link high sUA 


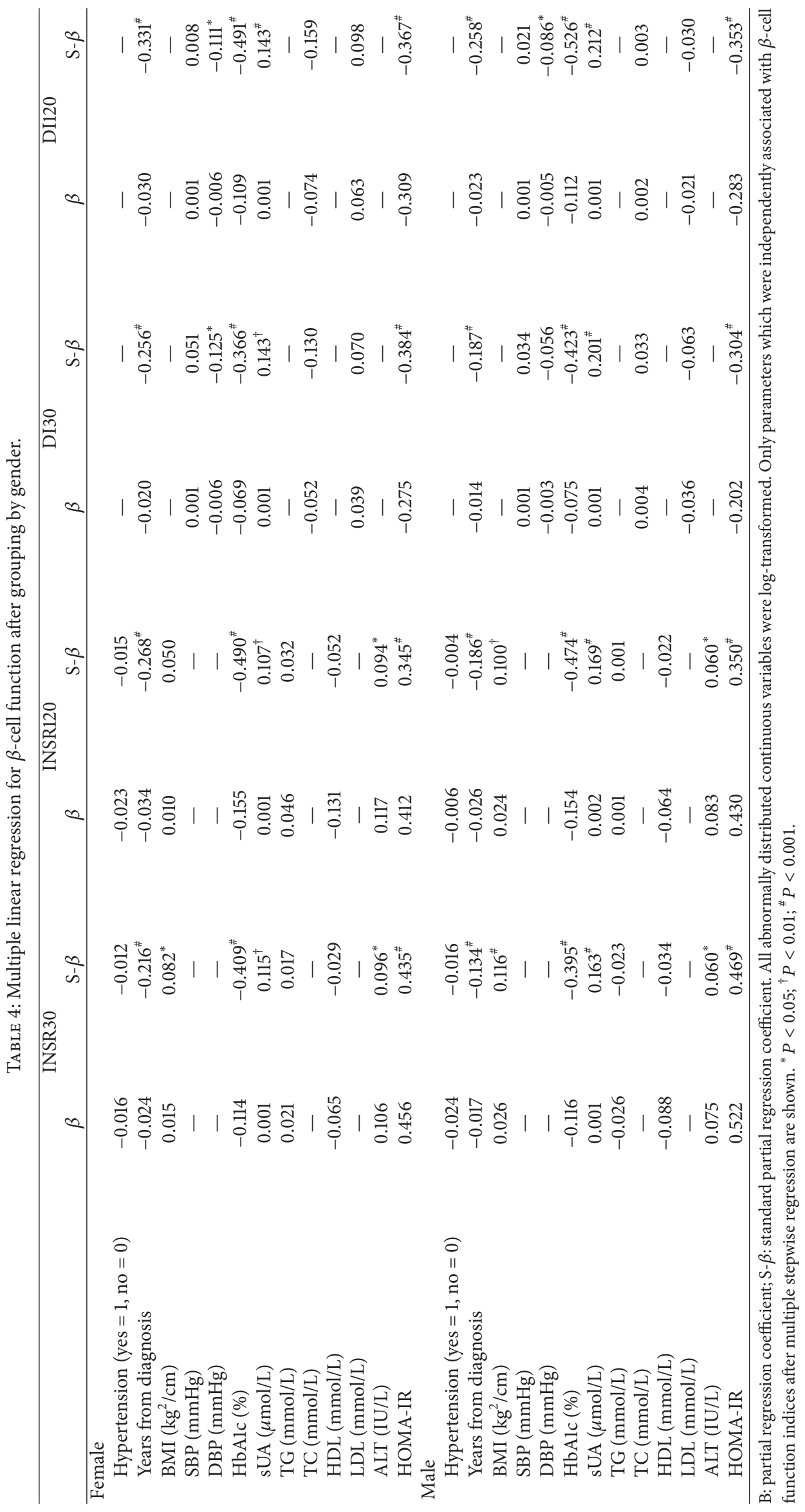




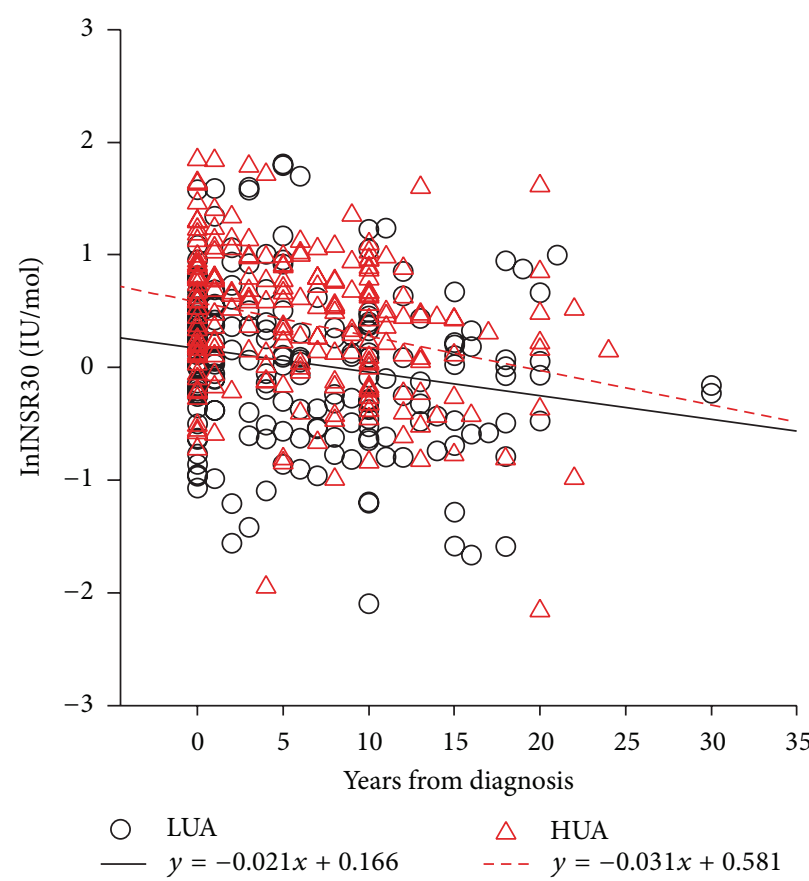

(a)

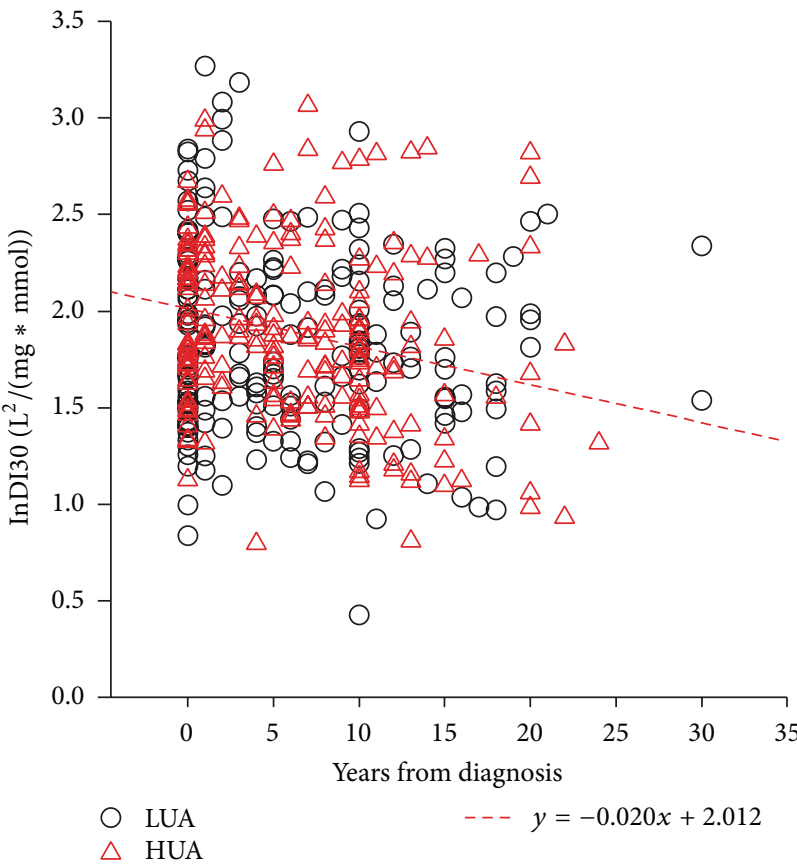

(c)

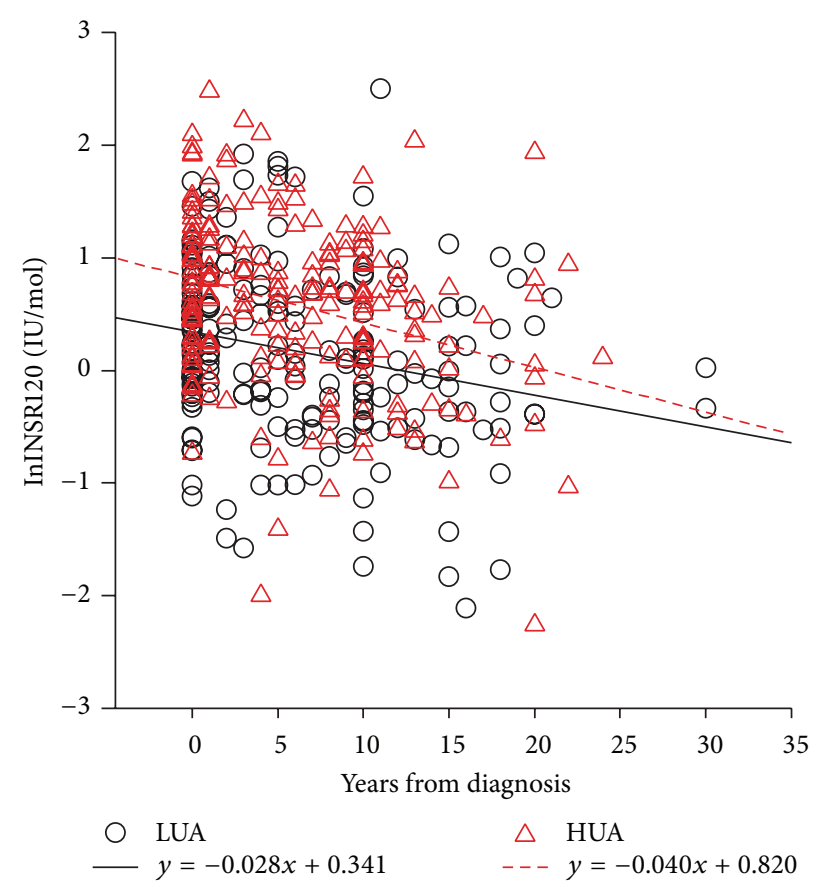

(b)

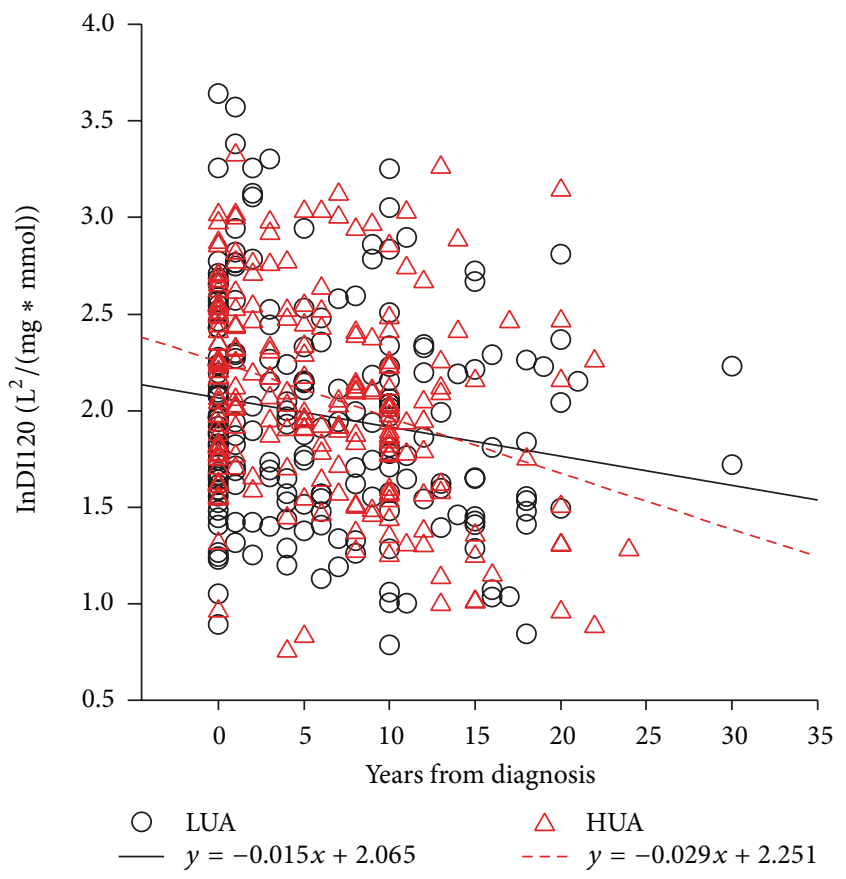

(d)

FIGURE 1: Scattered plots of simple linear regression between $\beta$-cell function and years from diagnosis in females. LUA, sUA levels under median of females; HUA, sUA levels above median of females. All $\beta$-cell function indices (INSR30, INSR30, DI30, and DI120) were logtransformed. Only significant regression lines and formulas are shown. All regression coefficients were negative. The decreases of INSR30, INSR120, and DI120 per year in the HUA group were greater than that in LUA group.

to insulin resistance and/or hyperinsulinaemia by increased activity of the hexose monophosphate shunt [22]. On the other hand, sUA not only may be a consequence of insulin resistance but also may actually promote or worsen insulin resistance. Specifically, a recent study showed that sUA plays an important role in the pathogenesis of MetS, possibly due to its ability to inhibit endothelial function. In detail, sUA has been shown to inhibit nitric oxide (NO) bioavailability and reduce NO concentration which is required in insulin stimulated glucose uptake $[23,24]$. Consequently, higher sUA 


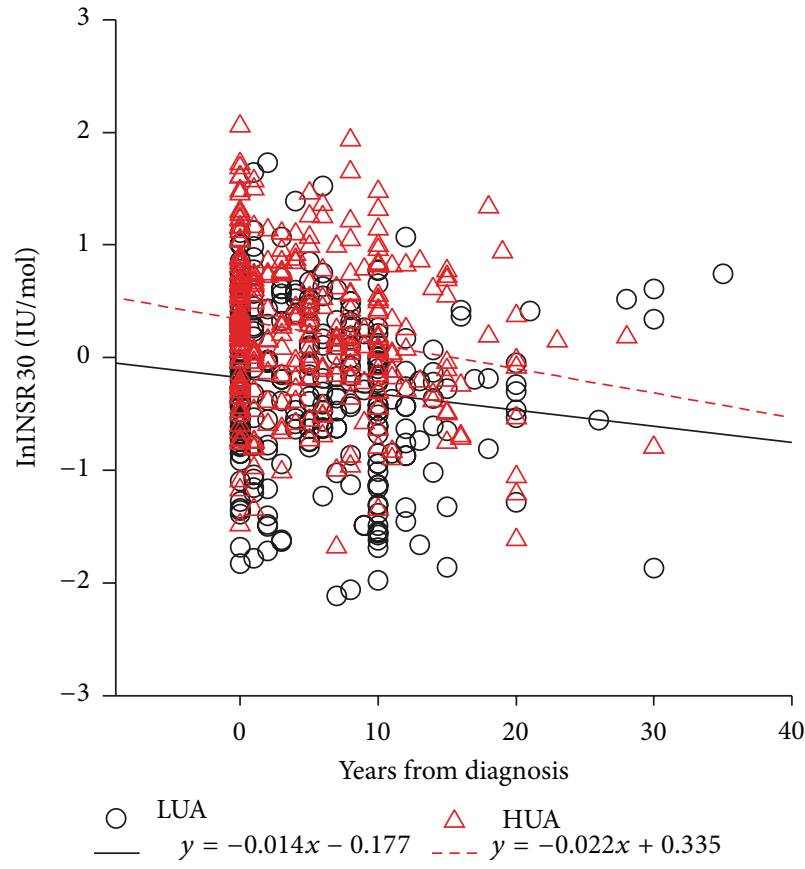

(a)

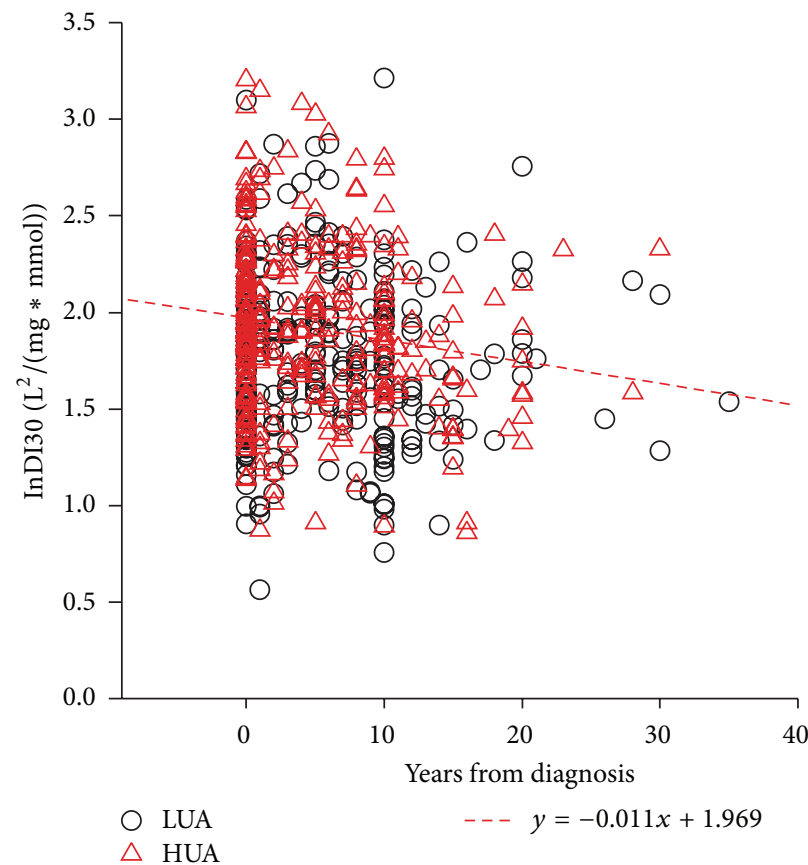

(c)

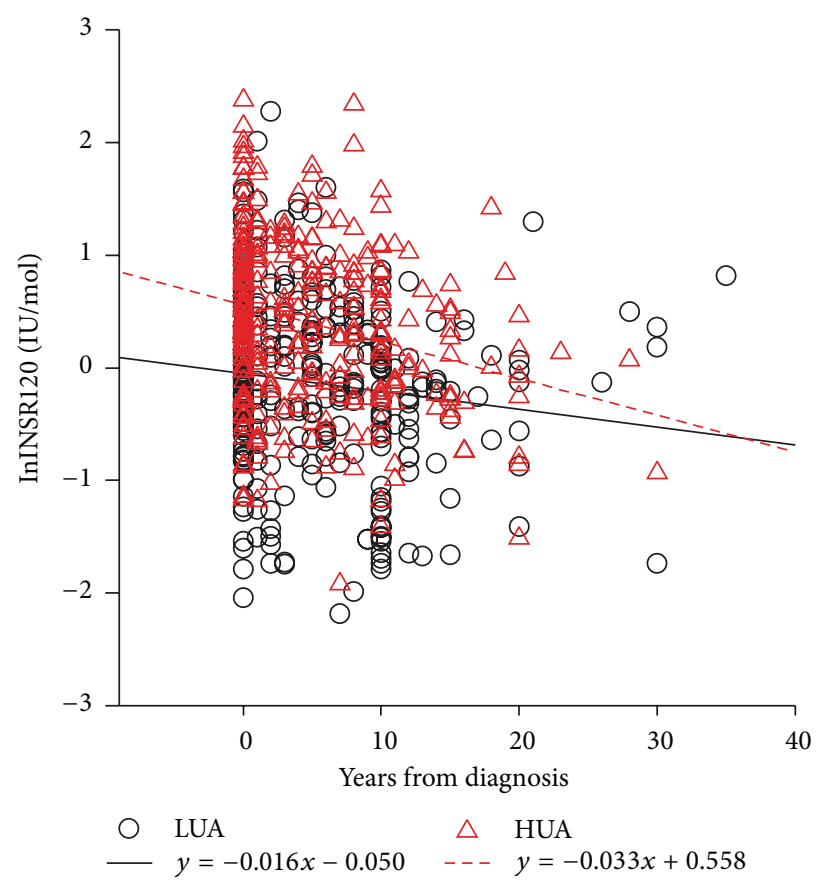

(b)

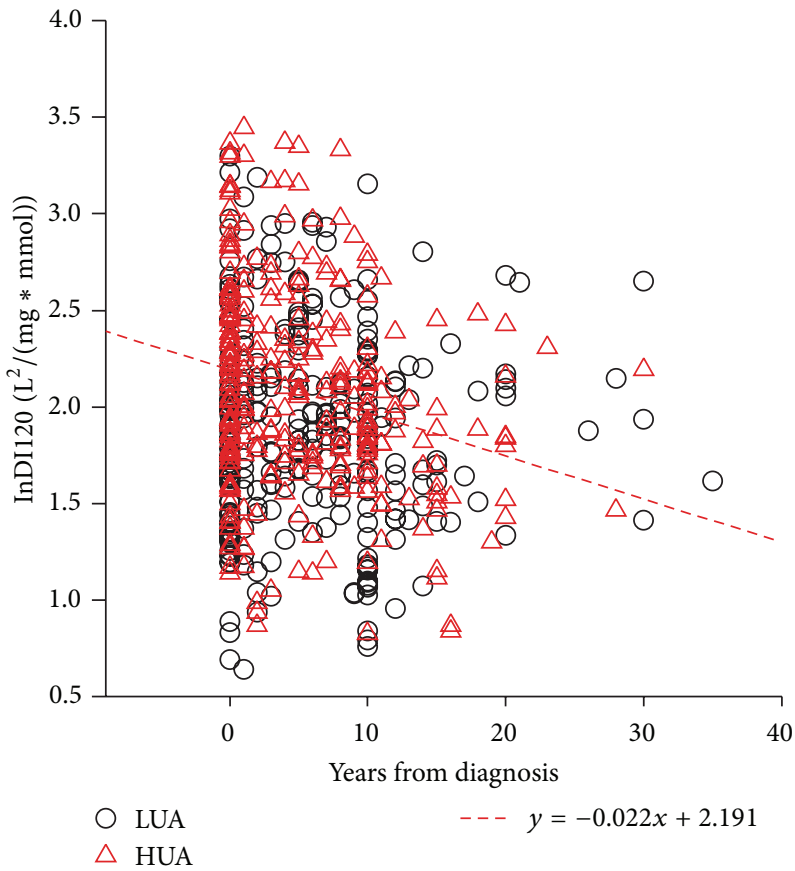

(d)

FIGURE 2: Scattered plots of simple linear regression between $\beta$-cell function and years from diagnosis in males. LUA, sUA levels under median of men; HUA, sUA levels above median of males. All $\beta$-cell function indices (INSR30, INSR30, DI30, and DI120) were log-transformed. Only significant regression lines and formulas are shown. All regression coefficients were negative. The decreases of INSR30 and INSR120 per year in the HUA group were greater than that in LUA group.

always keeps with more severe insulin resistance, that is, greater insulin demand of our organs.

Few previous researches investigated the interaction between $\beta$-cell function and sUA. In present study, we reported the correlation between sUA level and indices reflecting islet insulin secretion ability. Subjects with higher levels of sUA had higher insulin secretion, including the early phase (INSR30) and total (INSR120) insulin secretion, and after adjustment for variables associated with insulin resistance including BMI, TG, ALT, and HOMA-IR, sUA 
is still independently associated with insulin secretion. The reason why patients with type 2 diabetes of HUA group can secrete more insulin at the early stage was temporarily unknown. Insulin resistance may be one of the suppositional reasons, and the increased insulin secretion may be considered as a compensatory response to overcome the insulin resistance. Although subjects with higher sUA secrete more insulin, it does not mean that high sUA is beneficial to $\beta$-cell function. The insulin secretion ability in those seems to drop more rapidly than those having lower sUA as type 2 diabetes duration extends. At last, when the disease duration is long enough, the difference of both INSR30 and INSR120 between HUA and LUA groups diminishes. A recent study provided evidence that sUA has a direct negative effect on $\beta$-cell function, which could cause $\beta$-cell death and dysfunction by activation of the NF- $\kappa$ B and iNOSNO signal axis. This may partly explain the reason why insulin secretion ability seems to drop more rapidly in HUA group [25]. Nevertheless, the profound physiology changes connecting uric acid metabolism and insulin secretion is still worth further investigation.

Disposition indices which reflect the real glucose metabolism give attention to both insulin sensitivity and insulin secretion after glucose load. In our research, the sUA always kept positive association with disposition indices in total patients and after having been divided into gender groups. This finding is consistent with the result also got in our study that those subjects with higher sUA got statistically lower HbAlc level. All these suggest that high sUA level is associated with better glucose utilization. A mechanism underlying the relationship between glucose utilization and sUA levels may be due to the uricosuric effect of glycosuria, which means hyperglycemia facilitates uric acid excretion when the blood glucose level is above $10 \mathrm{mmol} / \mathrm{L} \mathrm{[26].} \mathrm{By}$ glycosuria, the sUA concentration of patients with high blood glucose levels may be low; however high blood glucose is harmful to islet $\beta$-cells. Furthermore, some studies demonstrated high sUA levels are associated with increased generation of free radicals and oxidative stress [27], which has various adverse effects on $\beta$-cell function $[28,29]$. This could be one possible reason for the faster decline of insulin secretion function in high sUA patients. However, other studies have suggested that sUA is an effective antioxidant $[30,31]$ and elevated sUA levels may reflect a compensatory mechanism contributing to the increased oxidative stress associated with the MetS. Collectively, the exact role of sUA in oxidation is still controversial, and further research is required.

We are very cautious about making conclusion in the close relation between sUA and $\beta$-cell insulin secretion function as well as glucose disposition because of two points: firstly, this is just a cross-sectional study rather than longitudinal study; secondly, subjects in our study received a wide diversity of hypoglycemic therapy which might influence the nature change of the islet function.

However, from this study, an independent positive association between sUA and $\beta$-cell function is confirmed, suggesting a potential close relation and interaction between uric acid and insulin secretion ability. Type 2 diabetic patients with higher sUA level have better insulin secretion but their residual $\beta$-cell function seems to decay more quickly.

\section{Conflict of Interests}

The authors declare that there is no conflict of interests regarding the publication of this paper.

\section{Acknowledgment}

The authors would like to thank Chong Shen, Department of Epidemiology and Biostatistics, School of Public Health, Nanjing Medical University, Nanjing, China, for statistical assistance.

\section{References}

[1] N. Ishizaka, Y. Ishizaka, E.-I. Toda, R. Nagai, and M. Yamakado, "Association between serum uric acid, metabolic syndrome, and carotid atherosclerosis in Japanese individuals," Arteriosclerosis, Thrombosis, and Vascular Biology, vol. 25, no. 5, pp. 1038-1044, 2005.

[2] I.-C. Hwang, S.-Y. Suh, A.-R. Suh, and H.-Y. Ahn, "The relationship between normal serum uric acid and nonalcoholic fatty liver disease," Journal of Korean Medical Science, vol. 26, no. 3, pp. 386-391, 2011.

[3] E. S. Ford, C. Li, S. Cook, and H. K. Choi, "Serum concentrations of uric acid and the metabolic syndrome among US children and adolescents," Circulation, vol. 115, no. 19, pp. 25262532, 2007.

[4] A. Dehghan, M. van Hoek, E. J. G. Sijbrands, A. Hofman, and J. C. M. Witteman, "High serum uric acid as a novel risk factor for type 2 diabetes," Diabetes Care, vol. 31, no. 2, pp. 361-362, 2008.

[5] K.-L. Chien, M.-F. Chen, H.-C. Hsu et al., "Plasma uric acid and the risk of type 2 diabetes in a Chinese community," Clinical Chemistry, vol. 54, no. 2, pp. 310-316, 2008.

[6] S. E. Kahn, "The relative contributions of insulin resistance and beta-cell dysfunction to the pathophysiology of Type 2 diabetes," Diabetologia, vol. 46, no. 1, pp. 3-19, 2003.

[7] F. Facchini, Y.-D. I. Chen, C. B. Hollenbeck, and G. M. Reaven, "Relationship between resistance to insulin-mediated glucose uptake, urinary uric acid clearance, and plasma uric acid concentration," Journal of the American Medical Association, vol. 266, no. 21, pp. 3008-3011, 1991.

[8] S. G. Tsouli, E. N. Liberopoulos, D. P. Mikhailidis, V. G. Athyros, and M. S. Elisaf, "Elevated serum uric acid levels in metabolic syndrome: an active component or an innocent bystander?" Metabolism: Clinical and Experimental, vol. 55, no. 10, pp. 12931301, 2006.

[9] American Diabetes Association, "Diagnosis and classification of diabetes mellitus," Diabetes Care, vol. 30, no. 1, supplement, pp. S42-S47, 2007.

[10] A. Stǎácaková, M. Javorský, T. Kuulasmaa, S. M. Haffner, J. Kuusisto, and M. Laakso, "Changes in insulin sensitivity and insulin release in relation to glycemia and glucose tolerance in 6,414 finnish men," Diabetes, vol. 58, no. 5, pp. 1212-1221, 2009.

[11] M. Matsuda and R. A. DeFronzo, "Insulin sensitivity indices obtained from oral glucose tolerance testing: comparison with the euglycemic insulin clamp," Diabetes Care, vol. 22, no. 9, pp. 1462-1470, 1999. 
[12] D. R. Matthews, J. P. Hosker, A. S. Rudenski et al., "Homeostasis model assessment: insulin resistance and $\beta$-cell function from fasting plasma glucose and insulin concentrations in man," Diabetologia, vol. 28, no. 7, pp. 412-419, 1985.

[13] R. Retnakaran, S. Shen, A. J. Hanley, V. Vuksan, J. K. Hamilton, and B. Zinman, "Hyperbolic relationship between insulin secretion and sensitivity on oral glucose tolerance test," Obesity, vol. 16, no. 8, pp. 1901-1907, 2008.

[14] C. Weyer, C. Bogardus, D. M. Mott, and R. E. Pratley, "The natural history of insulin secretory dysfunction and insulin resistance in the pathogenesis of type 2 diabetes mellitus," Journal of Clinical Investigation, vol. 104, no. 6, pp. 787-794, 1999.

[15] S. E. Kahn, "Clinical, review 135: the importance of $\beta$-cell failure in the development and progression of type 2 diabetes," Journal of Clinical Endocrinology and Metabolism, vol. 86, no. 9, pp. 4047-4058, 2001.

[16] United Kingdom Prospective Diabetes Study Group, "U.K. Prospective Diabetes Study 16. Overview of 6 years therapy of type II diabetes: a progressive disease," Diabetes, vol. 44, no. 11, pp. 1249-1258, 1995.

[17] S. del Prato, "Role of glucotoxicity and lipotoxicity in the pathophysiology of Type 2 diabetes mellitus and emerging treatment strategies," Diabetic Medicine, vol. 26, no. 12, pp.11851192, 2009.

[18] M. Y. Donath, D. J. Gross, E. Cerasi, and N. Kaiser, "Hyperglycemia-induced $\beta$-cell apoptosis in pancreatic islets of Psammomys obesus during development of diabetes," Diabetes, vol. 48, no. 4, pp. 738-744, 1999.

[19] K. Maedler, G. A. Spinas, D. Dyntar, W. Moritz, N. Kaiser, and M. Y. Donath, "Distinct effects of saturated and monounsaturated fatty acids on $\beta$-cell turnover and function," Diabetes, vol. 50, no. 1, pp. 69-76, 2001.

[20] H. K. Choi, D. B. Mount, and A. M. Reginato, "Pathogenesis of gout," Annals of Internal Medicine, vol. 143, no. 7, pp. 499-516, 2005.

[21] F. P. Cappuccio, P. Strazzullo, E. Farinaro, and M. Trevisan, "Uric acid metabolism and tubular sodium handling: results from a population-based study," Journal of the American Medical Association, vol. 270, no. 3, pp. 354-359, 1993.

[22] M. Modan, H. Halkin, A. Karasik, and A. Lusky, "Elevated serum uric acid-a facet of hyperinsulinaemia," Diabetologia, vol. 30, no. 9, pp. 713-718, 1987.

[23] T. Nakagawa, H. Hu, S. Zharikov et al., "A causal role for uric acid in fructose-induced metabolic syndrome," American Journal of Physiology. Renal Physiology, vol. 290, no. 3, pp. F625F631, 2006.

[24] S. Baldus, R. Köster, P. Chumley et al., "Oxypurinol improves coronary and peripheral endothelial function in patients with coronary artery disease," Free Radical Biology and Medicine, vol. 39, no. 9, pp. 1184-1190, 2005.

[25] L. Jia, J. Xing, Y. Ding et al., "Hyperuricemia causes pancreatic $\beta$-cell death and dysfunction through NF- $\kappa \mathrm{B}$ signaling pathway," PLoS ONE, vol. 8, no. 10, Article ID e78284, 2013.

[26] D. G. Cook, A. G. Shaper, D. S. Thelle, and T. P. Whitehead, "Serum uric acid, serum glucose and diabetes: relationships in a population study," Postgraduate Medical Journal, vol. 62, no. 733, pp. 1001-1006, 1986.

[27] J. Vásquez-Vivar, A. M. Santos, V. B. Junqueira, and O. Augusto, "Peroxynitrite-mediated formation of free radicals in human plasma: EPR detection of ascorbyl, albumin-thiyl and uric acidderived free radicals," Biochemical Journal, vol. 314, pp. 869-876, 1996.

[28] A. P. Robertson, "Chronic oxidative stress as a central mechanism for glucose toxicity in pancreatic islet beta cells in diabetes," Journal of Biological Chemistry, vol. 279, no. 41, pp. 42351-42354, 2004.

[29] Y. Zhang, T. Yamamoto, I. Hisatome et al., "Uric acid induces oxidative stress and growth inhibition by activating adenosine monophosphate-activated protein kinase and extracellular signal-regulated kinase signal pathways in pancreatic $\beta$ cells," Molecular and Cellular Endocrinology, vol. 375, no. 1-2, pp. 8996, 2013.

[30] F. J. Nieto, C. Iribarren, M. D. Gross, G. W. Comstock, and R. G. Cutler, "Uric acid and serum antioxidant capacity: a reaction to atherosclerosis?" Atherosclerosis, vol. 148, no. 1, pp. 131-139, 2000.

[31] K. Nyyssonen, E. Porkkala-Sarataho, and J. Kaikkonen, "Ascorbate and urate are the strongest determinants of plasma antioxidative capacity and serum lipid resistance to oxidation in Finnish men," Atherosclerosis, vol. 130, no. 1-2, pp. 223-233, 1997. 


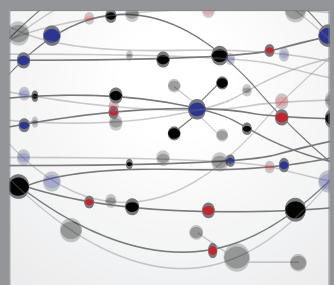

The Scientific World Journal
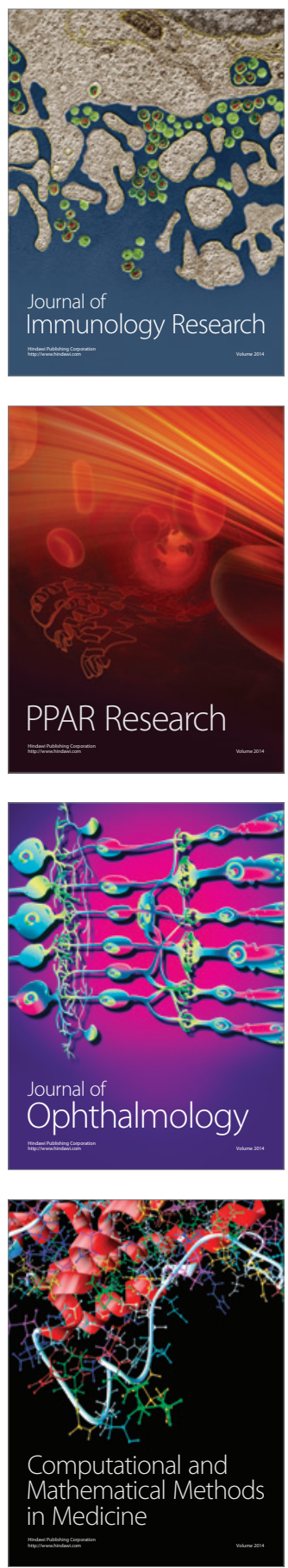

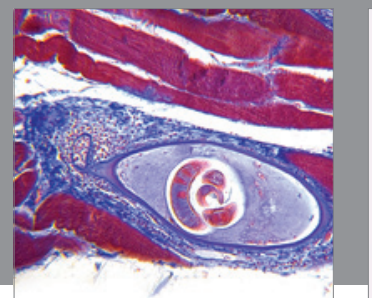

Gastroenterology

Research and Practice
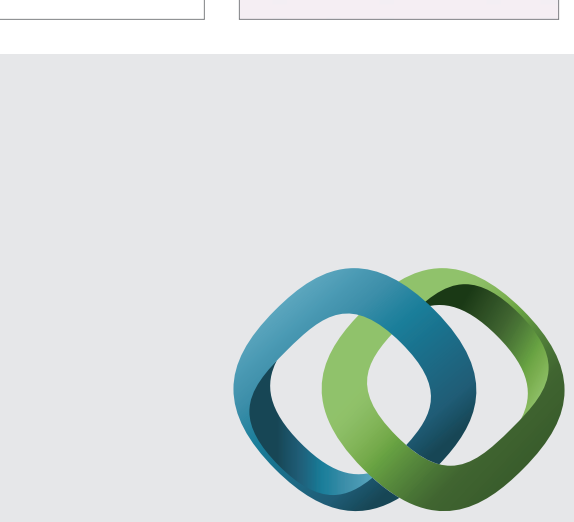

\section{Hindawi}

Submit your manuscripts at

http://www.hindawi.com
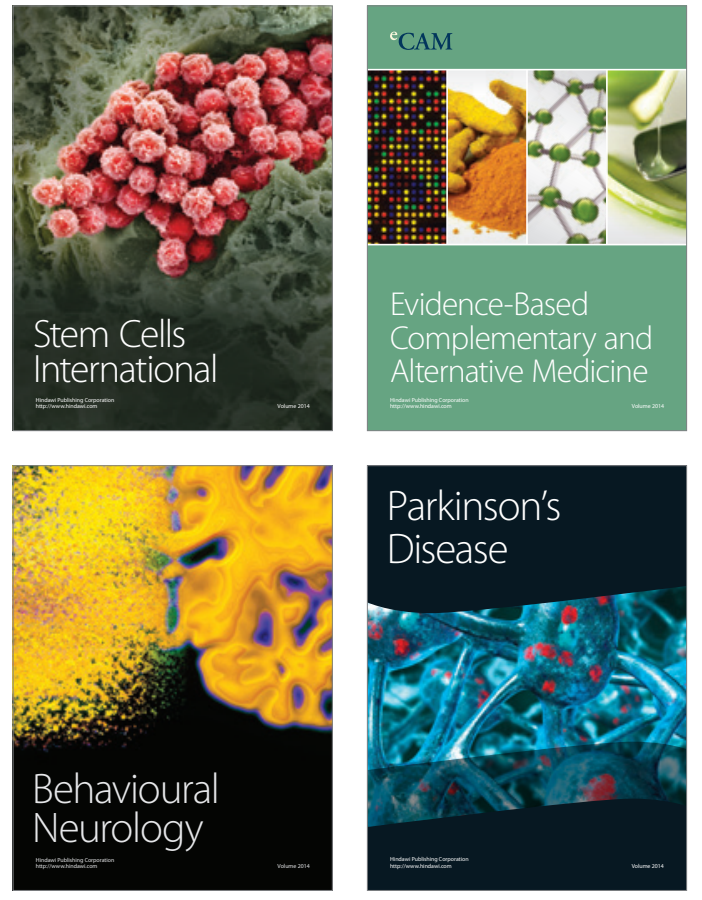
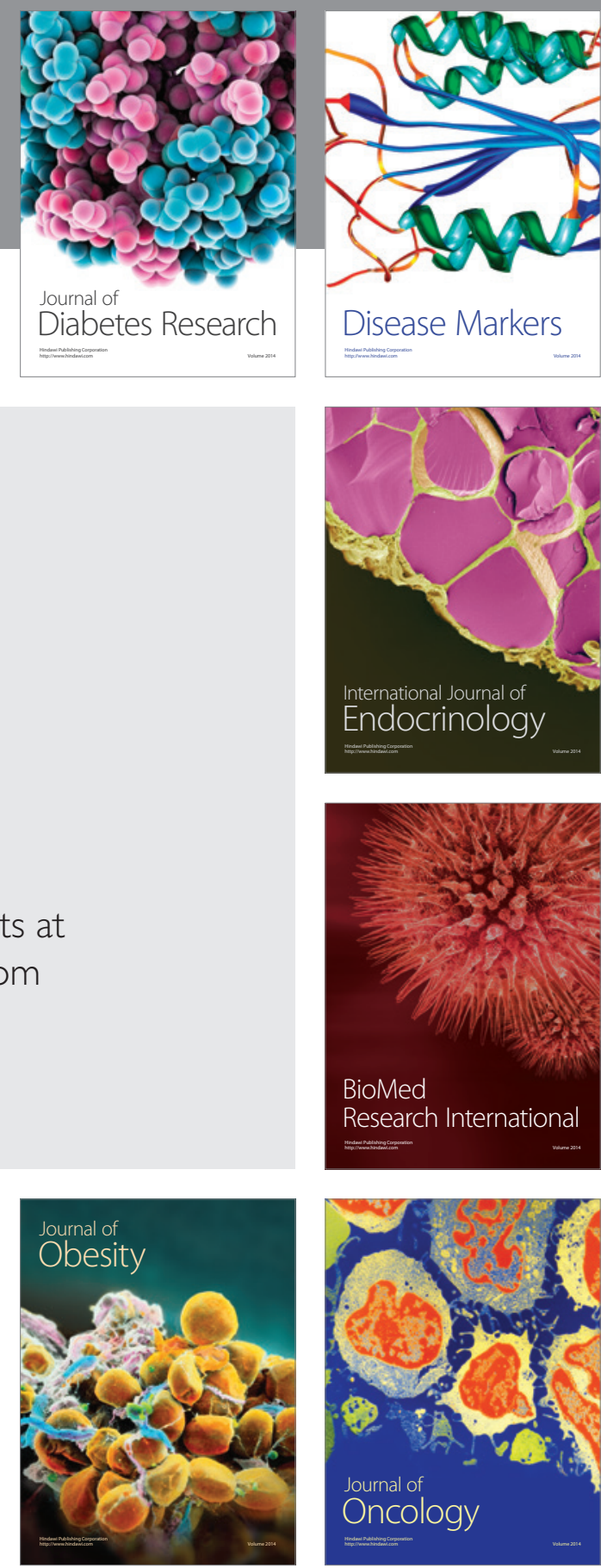

Disease Markers
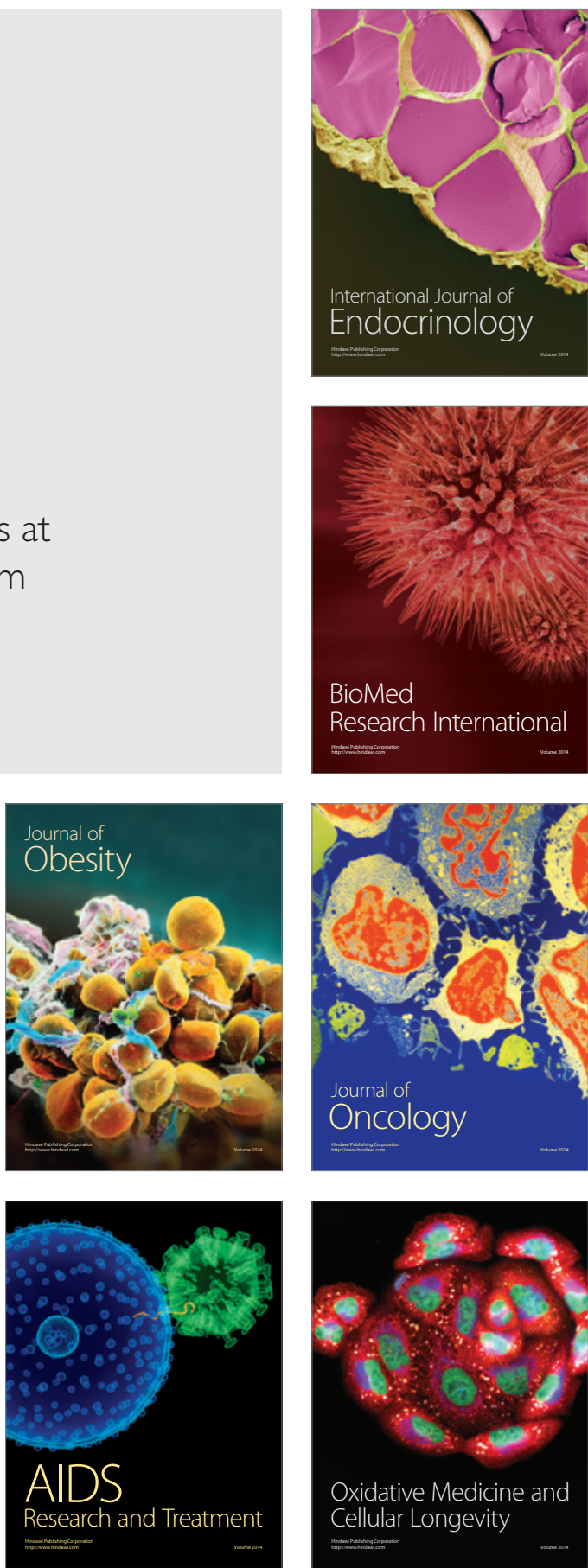